\title{
LAUE DIFFRACTION OF SPHERICAL MÖSSBAUER WAVES
}

\begin{abstract}
The symmetric Laue diffraction of Mössbauer rays is analyzed in the spherical-wave approximation. The saddle-point method is applied to calculate the $\gamma$-photon wave function within the Borrmann triangle in a thick crystal with strong nuclear absorption. Both the Rayleigh and resonant nuclear scatterings are taken into account. The interference oscillations of the diffracted beam intensity are shown to appear in the case of the Rayleigh scattering of Mössbauer radiation, which may be used for precision measurements of crystal parameters.

Ke ywords: Mössbauer effect, Laue diffraction, spherical wave, Borrmann triangle, $\gamma$-photon wave function.
\end{abstract}

Huge number of works has been devoted to the diffraction of x-rays and synchrotron radiation as well as Mössbauer radiation and matter waves (neutrons, electrons, etc.). In this way, a number of effects were discovered, among which the most striking were the pendellösung effect and the anomalous transmission of $\gamma$-photons and neutrons through a perfect crystal in the Laue (transmission) geometry. In the x-ray optics, the latter effect has been discovered by Borrmann [1-3]. The explanation of these phenomena has been given by the dynamical scattering theory [1-3] extended to the case of the resonant nuclear scattering by Kagan and Afanas'ev [4]. Its simplest version describes the case where single Bragg's condition is fulfilled. In such two-wave case, the incident plane electromagnetic wave generates two couples of waves inside the crystal, both of which are coherent superpositions of the transmitted and reflected waves. One of such couples has nodes at the scattering atoms and is therefore anomalously weakly absorbed, whereas another one strongly absorbed pare of the waves has antinodes there.

(c) A.YA. DZYUBLIK, V.YU. SPIVAK, 2016
The multiple scattering of x-ray photons by crystals is always described by the Maxwell equations [1-3]. In the same quasiclassical manner, Afanas'ev and Kagan [4] treated the resonant scattering of Mössbauer radiation by a perfect crystal. A more rigorous dynamical theory of the Mössbauer diffraction has been developed in the framework of the quantum electrodynamics by Hannon and Trammell [6, 7].

In typical Laue-diffraction experiments, the incident $\gamma$ quanta are first collimated by a slit, lying on the crystal surface and being parallel to the reflecting planes (see Fig. 1). The radiation transmitted through the entrance slit is spread in an angular region of the order of

$\sigma=\left(\overline{\Delta \theta^{2}}\right)^{1 / 2} \ll 1$

where $\Delta \theta=\theta-\theta_{\mathrm{B}}$ is the departure from Bragg's angle $\theta_{\mathrm{B}}$. Usually, the distribution width $\sigma$ for x-rays or Mössbauer rays is much larger than the angular interval, where the diffraction is still significant and which only amounts several angular seconds, which allows one to treat the task in the spherical wave approximation [7-10]. The collimated radiation spreads within the angular region from $-\theta_{\mathrm{B}}$ to $\theta_{\mathrm{B}}$, which forms the

ISSN 2071-0194. Ukr. J. Phys. 2016. Vol. 61, No. 9 
so-called Borrmann triangle. The intensity distribution of the transmitted and reflected radiation over the basis of the Borrmann triangle is analyzed with the aid of one more slit, which is also parallel to the reflecting crystal planes.

Kato [8-11] considered the incident x-ray spherical wave as a superposition of the classical electromagnetic plane waves, i.e., as a wave packet, spread over the angle $\theta$ about the Bragg angle $\theta_{\mathrm{B}}$. Every such plane component independently of one another are scattered by atoms of the crystal, forming the refracted and diffracted wave packets represented by the integrals over the angle $\theta$. Their diffraction was described in the two-wave approximation. Assuming the crystal thickness $D$ to be much larger than the pendellösung length $\Lambda_{\mathrm{L}}$, Kato [8-11] estimated these integrals over $\theta$ with the aid of the stationary-phase approximation. This method can be used only if the large parameter $D / \Lambda_{\mathrm{L}}$ is a real number. In other words, it can be applied only to weakly absorbing crystals.

At the same time, for the Mössbauer diffraction, when the resonant scattering amplitude of $\gamma$-quanta is already a complex number, the stationary-phase method is not applicable. In this case, such integrals should be estimated by the more general saddle-point method. To the best of our knowledge, so far there is no theory for the Mössbauer diffraction of spherical wave packets, spread over the energy and angle. Therefore, we will solve this task in the present paper. For simplicity, we consider the symmetric Laue case.

As was mentioned above, Hannon and Trammell $[6,7]$ described the $\gamma$-photons by the vector potential $\mathbf{A}(\mathbf{r}, t)$. Note, however, that the vector potential written in the momentum representation represents the photon wave function, while $\mathbf{A}(\mathbf{r}, t)$, as a function of time and coordinates, does not [12]. Bialynicki-Birula [13] and Cipe [14] (see also [15-19]) made break-through, having found that the photon wave function must be written in the form

$\boldsymbol{\Psi}(\mathbf{r}, t)=\sum_{\lambda= \pm 1} \int \frac{d \boldsymbol{\kappa}}{(2 \pi)^{3 / 2}} f_{\lambda}(\boldsymbol{\kappa}) \chi_{\kappa \lambda}(\mathbf{r}, t)$,

representing a superposition of photon plane waves

$\chi_{\kappa \lambda}(\mathbf{r}, t)=\mathbf{e}_{\lambda} \sqrt{\hbar \omega} e^{i \kappa \mathbf{r}-i \omega t}$

with the wave vector $\boldsymbol{\kappa}$, helicity $\lambda= \pm 1$, and frequency $\omega=c \kappa$. If the momentum wave function of a

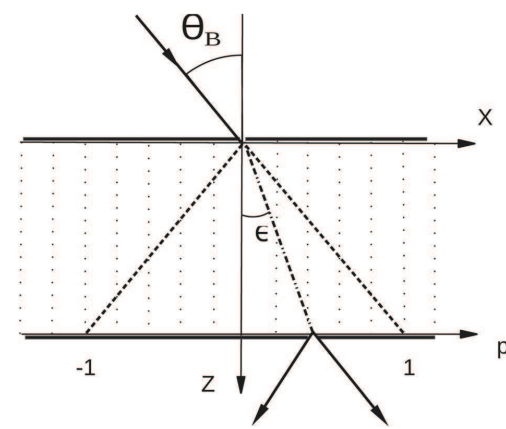

Fig. 1. Scheme of the Laue diffraction of the collimated Mössbauer radiation

photon $f_{\lambda}(\boldsymbol{\kappa})$ is normalized as

$\sum_{\lambda= \pm 1} \int d \boldsymbol{\kappa}\left|f_{\lambda}(\boldsymbol{\kappa})\right|^{2}=1$

then the squared modulus of $\boldsymbol{\Psi}(\mathbf{r}, t)$ determines the density of photon's mean energy at a given position $\mathbf{r}$ and the time $t$ [13-19]. Note that such a wave function was already employed in the quantum theory of the $\gamma$ photon transmission through vibrating crystals [20].

\section{Basic Formulas}

We choose the coordinate frame $x, y, z$, having the origin on the face crystal surface in the middle of the collimating slit, with the axis $z$ perpendicular to the crystal surface and directed inside the crystal and the axis $y$ along the slit. We consider here the symmetric Laue diffraction, when the axis $z$ is parallel to the reflecting planes. We suppose that all the incident rays propagate along the plane $x, z$ perpendicular to the slit, so that their wave vectors expressed in the spherical coordinates $\kappa, \theta, \varphi$ are given by

$\kappa \equiv \boldsymbol{\kappa}(\theta)=\{\kappa \sin \theta, 0, \kappa \cos \theta\}$.

In the general case, the polarizations of $\gamma$-quanta are mixed in the resonant scattering by nuclei. Below, we only consider the scattering by unpolarized Mössbauer nuclei with unsplt sublevels labeled by the magnetic quantum number. In this case, the mixing is avoided if $\gamma$-quanta have either $\pi$-polarization $\mathbf{e}_{s=1}$, lying in the scattering plane or $\sigma$-polarization $\mathbf{e}_{s=2}$, being perpendicular to this plane [21].

The frequency distribution for the phononless emission line is determined by the function

$G_{e}(\omega)=\left(\frac{\Gamma_{e}}{2 \pi \hbar}\right)^{1 / 2} \frac{e^{i \omega t_{0}}}{\omega-\omega_{0}+i \Gamma_{e} / 2 \hbar}$, 
where $\Gamma_{e}$ and $\hbar \omega_{0}$ are, respectively, the width and the energy of the excited level of the emitting nucleus. The function $G_{e}(\omega)$ is normalized to unity:

$$
\int_{-\infty}^{\infty} d \omega\left|G_{e}(\omega)\right|^{2}=1
$$

The angular dependence of the incident radiation can be described by the Gaussian function

$\mathcal{G}(\theta) \sim \exp \left[-\frac{(\Delta \theta)^{2}}{2 \sigma^{2}}\right]$

Usually, the dispersion $\sigma$ is much larger than the characteristic angle departure $\Delta \theta_{0}$, where the Bragg diffraction disappears. This fact enables us to use the spherical wave approximation, i.e., to replace $\mathcal{G}(\theta)$ by 1 .

Then the wave function of an incident $\gamma$-quantum with polarization $\mathbf{e}_{s}$ takes the form

$\Psi_{\text {in }}^{(s)}(\mathbf{r}, t)=\int_{-\infty}^{\infty} d \omega G_{e}(\omega) \int_{-\infty}^{\infty} d \theta \chi_{\boldsymbol{\kappa}(\theta) s}(\mathbf{r}, t)$.

The polarization vectors $\mathbf{e}_{s}(\boldsymbol{\kappa})$ are perpendicular to the wave vector $\boldsymbol{\kappa}$ and, therefore, depend also on the angle $\theta$. However, one can neglect this dependence due to the inequality $\sigma \ll 1$.

The time dependence of a $\gamma$-quantum incident at the face surface $(z=0)$ is described by the exponential:

$\left|\boldsymbol{\Psi}_{\text {in }}^{(s)}(0, t)\right|^{2} \sim \exp \left[-\Gamma_{e}\left(t-t_{0}\right) / \hbar\right] \theta\left(t-t_{0}\right)$,

where

$\theta(x)= \begin{cases}1, & x>0 \\ 0, & x<0\end{cases}$

is the Heaviside step function. According to (10), $t_{0}$ means the moment of photon's arrival at the crystal.

The coherent scattering amplitude of $\gamma$-quanta by the $j$-th nucleus with unsplit sublevels is given by [21]

$$
\begin{aligned}
& f_{\mathrm{coh}}\left(\boldsymbol{\kappa}, \mathbf{e}_{s} ; \boldsymbol{\kappa}^{\prime}, \mathbf{e}_{s}^{\prime}\right)_{j}^{N}=-c_{0}\left(\frac{2 I_{e}+1}{2 I_{g}+1}\right) \times \\
& \times \frac{1}{4 \kappa} \frac{\Gamma_{\gamma} e^{-W_{j}(\boldsymbol{\kappa})-W_{j}\left(\boldsymbol{\kappa}^{\prime}\right)}}{\hbar\left(\omega-\omega_{0}^{\prime}\right)+i \Gamma / 2} P_{s}^{N},
\end{aligned}
$$

where $c_{0}$ is the relative concentration of the Mössbauer isotope, $I_{e}$ and $I_{g}$ are the nuclear spins in the excited and ground states, $e^{-W_{j}(\mathbf{k})}$ is the LambMössbauer factor, $P_{s}^{N}$ is the nuclear polarization factor, $\Gamma$ and $\Gamma_{\gamma}$ are, respectively, the total and radiative widths of the resonant nuclear level with the energy $\hbar \omega_{0}^{\prime}$. In the case of M1 transitions, $P_{s}^{N}=1$ for the $\pi$-polarization, and $P_{s}^{N}=\mathbf{e} \cdot \mathbf{e}^{\prime}$ for the $\sigma$-polarization [21].

The coherent Rayleigh scattering amplitude by the $j$ th atom is determined by the expression

$f_{\mathrm{coh}}\left(\boldsymbol{\kappa}, \mathbf{e}_{s} ; \boldsymbol{\kappa}^{\prime}, \mathbf{e}_{s}^{\prime}\right)_{j}^{R}=e^{-W_{j}(\mathbf{Q})} r_{0} F_{e}^{(j)}(\mathbf{Q})\left(\mathbf{e}_{s} \cdot \mathbf{e}_{s}^{\prime *}\right)+$

$+i(\kappa / 4 \pi) \sigma_{e}^{(j)}$

where the form-factor of the $j$ th atom

$F_{e}^{(j)}(\mathbf{Q})=\int \varrho_{e}^{(j)}(\mathbf{r}) e^{i \mathbf{Q r}} d \mathbf{r}$

$\mathbf{Q}=\boldsymbol{\kappa}-\boldsymbol{\kappa}^{\prime}$ is the scattering vector, $\varrho_{e}^{(j)}(\mathbf{r})$ is the density of atomic electrons, $r_{0}=e^{2} / m c^{2}$ denotes the classical radius of an electron, $\sigma_{e}^{(j)}$ is the absorption cross section of $\gamma$-quanta by electrons of the $j$ th atom.

The coherent scattering amplitude of $\gamma$-quanta by an elementary cell of the crystal reads

$F_{\text {coh }}\left(\boldsymbol{\kappa}, \mathbf{e} ; \boldsymbol{\kappa}^{\prime}, \mathbf{e}^{\prime}\right)=F_{\text {coh }}\left(\boldsymbol{\kappa}, \mathbf{e} ; \boldsymbol{\kappa}^{\prime}, \mathbf{e}^{\prime}\right)^{N}+$

$+F_{\mathrm{coh}}\left(\boldsymbol{\kappa}, \mathbf{e} ; \boldsymbol{\kappa}^{\prime}, \mathbf{e}^{\prime}\right)^{R}$,

where

$F_{\text {coh }}\left(\boldsymbol{\kappa}, \mathbf{e} ; \boldsymbol{\kappa}^{\prime}, \mathbf{e}^{\prime}\right)^{N(R)}=\sum_{j} e^{i \mathbf{Q} \boldsymbol{\rho}_{j}} \times$

are the nuclear and Rayleigh coherent scattering amplitudes depending on the radius-vector $\boldsymbol{\rho}_{j}$ for the equilibrium position of the $j$-th atom in the elementary cell.

\section{Dynamical Scattering Theory}

Every incident plane wave $\chi_{\kappa s}(\mathbf{r}, t)$ of the wave packet (8) is scattered independently of one another by atoms of the crystal bearing the wave $\boldsymbol{\psi}_{\boldsymbol{\kappa} s}(\mathbf{r}, t)$. In other words, the complete wave function of the photon will be

$\boldsymbol{\Psi}_{s}(\mathbf{r}, t)=\int_{-\infty}^{\infty} d \omega G_{e}(\omega) \int_{-\infty}^{\infty} d \theta \boldsymbol{\psi}_{\kappa s}(\mathbf{r}, t)$.

In the two-wave case, the wave vectors of the refracted and diffracted waves inside the crystal are

ISSN 2071-0194. Ukr. J. Phys. 2016. Vol. 61, No. 9 
$\mathbf{k}(\theta)$ and $\mathbf{k}_{1}(\theta)=\mathbf{k}(\theta)+\boldsymbol{\tau}_{1}$, respectively. Here, $\boldsymbol{\tau}_{1}$ denotes a reciprocal lattice vector divided by $2 \pi$. The components of the vectors $\mathbf{k}(\theta)$ and $\boldsymbol{\kappa}(\theta)$ along the face surface $z=0$ coincide. Therefore, the vector $\mathbf{k}(\theta)$ can be written as

$\mathbf{k}(\theta)=\boldsymbol{\kappa}(\theta)+\delta(\theta) \mathbf{n}$,

where $\mathbf{n}$ is the unit vector along the axis $z$. Note that the parameter $\delta(\theta)=k_{z}(\theta)-\kappa_{z}(\theta)$ depends in addition on the wave number $\kappa$ of the $\gamma$-quantum in vacuum. For brevity, this dependence is not written down.

Then the wave function $\boldsymbol{\psi}_{\boldsymbol{\kappa} s}(\mathbf{r}, t)$ inside the crystal is

$$
\begin{aligned}
& \boldsymbol{\psi}_{\boldsymbol{\kappa} s}(\mathbf{r}, t)=\sum_{\nu=0,1} \mathbf{e}_{\nu s} \sqrt{\hbar \omega} \times \\
& \times \sum_{\iota=1,2} C_{\nu s}^{(\iota)}(\theta) e^{i \boldsymbol{\kappa}_{\nu}(\theta) \mathbf{r}+i \delta_{\iota s}(\theta) z-i \omega t} .
\end{aligned}
$$

Note that all the wave parameters written above depend still on the frequency $\omega$.

The amplitudes $C$ and the wave vectors $\mathbf{k}$ are determined by the algebraic equations [4]

$$
\begin{aligned}
& \left(k^{2}(\theta) / \kappa^{2}(\theta)-1\right) C_{0 s}=g_{00}^{(s)} C_{0 s}+g_{01}^{(s)} C_{1 s}, \\
& \left(k_{1}^{2}(\theta) / \kappa^{2}(\theta)-1\right) C_{1 s}=g_{10}^{(s)} C_{0 s}+g_{11}^{(s)} C_{1 s} .
\end{aligned}
$$

The scattering matrix $g_{\mu \nu}^{(s)}$ is defined by the expression

$g_{\mu \nu}^{(s)}=\frac{4 \pi}{\kappa^{2} v_{0}} F_{\nu \mu}^{(s)}, \quad \mu, \nu=0,1$,

where $v_{0}$ stands for the volume of the elementary cell and the abbreviation $F_{\nu \mu}^{(s)}=F_{\mathrm{coh}}\left(\boldsymbol{\kappa}_{\nu}, \mathbf{e}_{\nu s} ; \boldsymbol{\kappa}_{\mu}, \mathbf{e}_{\mu s}\right)$ is used together with the wave vectors $\boldsymbol{\kappa}_{0}=\boldsymbol{\kappa}\left(\theta_{\mathrm{B}}\right)$ and $\kappa_{1}=\kappa_{0}+\tau_{1}$.

The solution of Eqs. (20) in the case of symmetric Laue diffraction is

$$
\begin{aligned}
& \delta_{\iota s}(\theta)=\frac{\kappa}{2 \gamma_{0}}\left[g_{00}^{(s)}+\sin \left(2 \theta_{\mathrm{B}}\right) \Delta \theta+\right. \\
& \left.+(-1)^{\iota+1} \sqrt{g_{01}^{(s)} g_{10}^{(s)}+\sin ^{2}\left(2 \theta_{\mathrm{B}}\right)(\Delta \theta)^{2}}\right]
\end{aligned}
$$

where $\iota=1,2$ and $\gamma_{0}=\cos \theta_{\mathrm{B}}$.

Introducing the deviation parameter

$\eta=\frac{\sin 2 \theta_{\mathrm{B}}}{\sqrt{g_{01}^{(s)} g_{10}^{(s)}}} \Delta \theta$, we rewrite $(22)$ as

$\delta_{\iota s}=\frac{\kappa g_{00}^{(s)}}{2 \gamma_{0}}+\frac{\pi}{\Lambda_{\mathrm{L}}}\left(\eta+(-1)^{\iota+1} \sqrt{1+\eta^{2}}\right)$,

where

$\Lambda_{\mathrm{L}}=\frac{2 \pi \gamma_{0}}{\kappa \sqrt{g_{01}^{(s)} g_{10}^{(s)}}}$.

In the case of the x-ray scattering in weakly absorbing crystals, the parameter $\Lambda_{\mathrm{L}}$ means the pendellösung distance, defining the period of the intensity swings between the diffracted and refracted beams (see also $[2])$. But, in a close vicinity of the nuclear resonance, where $\operatorname{Re} \Lambda_{\mathrm{L}} \sim \operatorname{Im} \Lambda_{\mathrm{L}}$, this parameter loses such a simple interpretation.

The amplitudes of the waves satisfy the following boundary conditions at the entrance surface of the crystal:

$\sum_{\iota=1,2} C_{0 s}^{(\iota)}(\eta)=1, \quad \sum_{\iota=1,2} C_{1 s}^{(\iota)}(\eta)=0$.

From Eqs. (20) and (26), one has

$$
\begin{aligned}
& C_{0 s}^{(\iota)}(\eta)=\frac{1}{2}\left(1+(-1)^{\iota} \frac{\eta}{\sqrt{1+\eta^{2}}}\right) \\
& C_{1 s}^{(\iota)}(\eta)=\frac{(-1)^{\iota+1}}{2}\left(\frac{g_{10}^{(s)}}{g_{01}^{(s)}}\right)^{1 / 2} \frac{1}{\sqrt{1+\eta^{2}}} .
\end{aligned}
$$

The intensity distribution of $\gamma$-photons over the basis of the Borrmann triangle is analyzed with the aid of the scanning slit located on the rear surface and also directed along the axis $y$. The position of such a slit is determined by the dimensionless parameter

$p=\tan \epsilon / \tan \theta_{\mathrm{B}}$

where $\tan \epsilon=x_{a} / D$, and $x_{a}$ is the usual coordinate of the analyzing slit [1].

In order to get the refracted and diffracted waves, we substitute (19) into (17) and expand the vectors $\boldsymbol{\kappa}$ of the exponential $e^{i \boldsymbol{\kappa} \mathbf{r}}$ in $\Delta \theta$, by keeping only the linear terms. In addition, we introduce the designations

$N=\frac{\pi D}{\left|\Lambda_{\mathrm{L}}\right|}$

and

$S_{\iota}(\eta)=i\left(\left|\Lambda_{\mathrm{L}}\right| / \Lambda_{\mathrm{L}}\right)\left[p \eta+(-1)^{\iota+1} \sqrt{1+\eta^{2}}\right]$. 
Then, behind the crystal, the waves are

$\boldsymbol{\Psi}_{\nu s}(\mathbf{r}, t)=\int_{-\infty}^{\infty} d \omega G_{e}(\omega) \frac{\sqrt{g_{01}^{(s)} g_{10}^{(s)}}}{\sin 2 \theta_{\mathrm{B}}} \boldsymbol{\Phi}_{\nu s}(\omega ; \mathbf{r}, t)$

where the function

$$
\begin{aligned}
& \boldsymbol{\Phi}_{\nu s}(\omega ; \mathbf{r}, t)=\int_{-\infty}^{\infty} d \eta \times \\
& \times \sum_{\iota=1,2} C_{\nu s}^{(\iota)}(\eta) e^{N S_{\iota}(\eta)} \chi_{\boldsymbol{\kappa}_{\nu s}}(\mathbf{r}, t)
\end{aligned}
$$

describes the wave packet with fixed frequency $\omega=$ $=\kappa c$, and the wave vectors $\boldsymbol{\kappa}_{\nu}=\boldsymbol{\kappa}_{\nu}\left(\theta_{\mathrm{B}}\right)$, indicating the direction of propagation.

For a thick crystal as $N \gg 1$, one can estimate the integral over $\eta$ with the aid of the saddle-point method (see, e.g., [22]). By equating the derivative $S_{\iota}^{\prime}(\eta)$ to zero, one finds the saddle point for the $\iota$-th branch:

$\eta_{0}^{(\iota)}=(-1)^{\iota} \frac{p}{\sqrt{1-p^{2}}}$.

After that the integration contour is displaced to the complex plane $\eta=\eta_{r}+i \eta_{i}$, where $\eta_{r}$ and $\eta_{i}$ represent the real and imaginary parts of $\eta$. This contour should cross the $\iota$-th saddle point along the direct line, where $\operatorname{Im} S_{\iota}(\eta)=$ const, which provides a steepest descent of the function $S_{\iota}(\eta)$. Such a line is declined with respect to the real axis $\eta_{r}$ by the angle

$\vartheta_{\iota}=\frac{\pi}{2}-\frac{1}{2} \arg S_{\iota}^{\prime \prime}\left(\eta_{0}\right)$,

where the second derivative of $S_{\iota}(\eta)$ at the saddle point is

$S_{\iota}^{\prime \prime}\left(\eta_{0}\right)=i(-1)^{\iota+1}\left(\frac{\left|\Lambda_{\mathrm{L}}\right|}{\Lambda_{\mathrm{L}}}\right)\left(1-p^{2}\right)^{3 / 2}$.

The substitution of (35) into (34) at $|p|<1$ gives

$\vartheta_{\iota}=(-1)^{\iota+1} \frac{\pi}{4}+\frac{1}{2} \arg \Lambda_{\mathrm{L}}$.

For $|p|>1$, one should add $\pi / 4$ to this expression.

The saddle-point estimation of integral (32) results in

$$
\mathbf{\Phi}_{\nu s}(\omega ; \mathbf{r}, t)=\sum_{\iota=1,2} C_{\nu}^{(\iota)}\left(\eta_{0}\right) e^{N S_{\iota}\left(\eta_{0}\right)} \times
$$

$\times \sqrt{\frac{2 \pi}{N\left|S_{\iota}^{\prime \prime}\left(\eta_{0}\right)\right|}} e^{i \vartheta_{\iota}} \chi_{\kappa s}(\mathbf{r}, t)$.

It is convenient to introduce the dimensionless parameters

$$
\begin{aligned}
& x=\frac{2 \hbar\left(\omega-\omega_{0}^{\prime}\right)}{\Gamma}, \Delta x_{0}=\frac{2 \hbar\left(\omega_{0}-\omega_{0}^{\prime}\right)}{\Gamma}, \\
& \tilde{\Gamma}_{e}=\frac{\Gamma_{e}}{\Gamma}
\end{aligned}
$$

and the function

$\zeta(x)=\frac{\pi D}{\Lambda_{\mathrm{L}}(x)} \sqrt{1-p^{2}}$.

By using all these definitions and incorporating all the above formulas in Eq. (37), one gets the $\gamma$-photon wave function beside the detector separated at the distance $R$ from the analyzing slit, having the coordinate $x_{a}$. For the diffracted wave function up to an unessential factor $\exp \left(\kappa \sin \theta_{\mathrm{B}} x_{a}\right)$, we obtain the expression

$$
\begin{aligned}
& \boldsymbol{\Psi}_{1 s}(\mathbf{r}, t)=\int_{-\infty}^{\infty} d x \frac{e^{-i x t^{*} / 2 \tau_{N}}}{x-\Delta x_{0}+i \tilde{\Gamma}_{e}} \frac{1}{\left(1-p^{2}\right)^{1 / 4}} \times \\
& \times\left\{\exp \left[-i\left(\zeta(x)+\frac{\pi}{4}\right)\right]-\exp \left[i\left(\zeta(x)+\frac{\pi}{4}\right)\right]\right\} \times \\
& \times B_{1 s}(x) \mathbf{e}_{1 s} \sqrt{\hbar \omega_{0}^{\prime}} e^{-i \omega_{0}^{\prime} t^{*}}
\end{aligned}
$$

where

$t^{*}=t-t_{0}-R / c$

means the retarded time, $\tau_{N}=\hbar / \Gamma$ the nuclear lifetime,

$B_{1 s}(x)=\frac{g_{10}^{(s)}(x)}{2 \sin 2 \theta_{\mathrm{B}}} \times$
$\times \sqrt{\frac{\Gamma_{e}}{\hbar}}\left(\frac{2 \Lambda_{\mathrm{L}}(x)}{D}\right)^{1 / 2} \exp \left[\frac{i \kappa D g_{00}^{(s)}(x)}{2 \gamma_{0}}\right]$.

As to the refracted wave, it is given by

$$
\begin{aligned}
& \mathbf{\Psi}_{0 s}(\mathbf{r}, t)=\int_{-\infty}^{\infty} d x \frac{e^{-i x t^{*} / 2 \tau_{N}}}{x-\Delta x_{0}+i \tilde{\Gamma}_{e}} \frac{1+p}{\left(1-p^{2}\right)^{3 / 4}} \times \\
& \times\left\{\exp \left[-i\left(\zeta(x)+\frac{\pi}{4}\right)\right]+\exp \left[i\left(\zeta(x)+\frac{\pi}{4}\right)\right]\right\} \times \\
& \times B_{0 s}(x) \mathbf{e}_{0 s} \sqrt{\hbar \omega_{0}^{\prime}} e^{-i \omega_{0}^{\prime} t^{*}},
\end{aligned}
$$


where

$B_{0 s}(x)=\left(\frac{g_{01}^{(s)}(x)}{g_{10}^{(s)}(x)}\right)^{1 / 2} B_{1 s}(x)$.

Inequalities (1) along with $\Gamma_{e} \ll \hbar \omega_{0}$ allow us to replace the function $\sqrt{\hbar \omega}$ in the integrals over $\omega$ by $\sqrt{\hbar \omega_{0}}$.

Inside the Borrmann triangle, $-1<p<1$, the diffracted beam intensity is

$I_{1 s}(p) \sim \frac{1}{\sqrt{1-p^{2}}} \int_{-\infty}^{\infty} d x \frac{e^{-\mu(x) D / \gamma_{0}}}{\left[\left(x-\Delta x_{0}\right)^{2}+\tilde{\Gamma}_{e}^{2}\right]} \times$
$\times\left|\frac{g_{10}^{(s)}(x)}{g_{01}^{(s)}(x)}\right|\left\{\sinh ^{2}\left[\zeta_{i}(x)\right]+\sin ^{2}\left[\zeta_{r}(x)+\frac{\pi}{4}\right]\right\}$,

and the refracted one is

$$
\begin{aligned}
& I_{0 s}(p) \sim \frac{1}{\sqrt{1-p^{2}}}\left(\frac{1+p}{1-p}\right) \times \\
& \times \int_{-\infty}^{\infty} d x \frac{e^{-\mu(x) D / \gamma_{0}}}{\left[\left(x-\Delta x_{0}\right)^{2}+\tilde{\Gamma}_{e}^{2}\right]} \times \\
& \times\left\{\sinh ^{2}\left[\zeta_{i}(x)\right]+\sin ^{2}\left[\zeta_{r}(x)-\frac{\pi}{4}\right]\right\},
\end{aligned}
$$

where $\zeta_{r}(x)$ and $\zeta_{i}(x)$ denote the real and imaginary parts of the complex function $\zeta(x)$, and

$\mu(x)=\kappa \operatorname{Im} g_{00}^{(s)}(x)$

stands for the absorption coefficient of $\gamma$-quanta far from the Bragg condition. Recall that the function $\zeta(x)$ defined by Eq. (39) contains the factor $\sqrt{1-p^{2}}$. Note also that, strictly speaking, the product $N \sqrt{1-p^{2}}$ serves, rather than $N$, as a large parameter in the saddle-point approximation. Therefore, the derived formulas cannot be applied at $|p| \approx 1$.

Some simplifications of the formulas appear, when the elementary cell contains a single Mössbauer atom. Then the transmission of $\gamma$-quanta is usually specified by the nuclear thickness parameter

$\beta=\sigma_{0} e^{-2 W(\kappa)} n_{0}$

depending on the resonant value of absorption cross section

$\sigma_{0}=\frac{2 \pi}{\kappa^{2}}\left(\frac{2 I_{e}+1}{2 I_{g}+1}\right) \frac{\Gamma_{\gamma}}{\Gamma}$,

ISSN 2071-0194. Ukr. J. Phys. 2016. Vol. 61, No. 9 and the number of resonant nuclei per unit square of the absorber $n_{0}=c_{0} D / v_{0}$. We assume also that the vibrations of atoms in the crystal are isotropic, so that the Lamb-Mössbauer factor $e^{-W(\boldsymbol{\kappa})}$ does not depend on the orientation of the wave vector $\boldsymbol{\kappa}$. All this enables us to rewrite the constituents of the function $\zeta(x)=\zeta_{r}(x)+i \zeta_{i}(x)$ as

$\zeta_{r}(x)=\left[-\frac{\beta}{2} \frac{x}{1+x^{2}} P_{N}+\left(\frac{2 \pi D}{\kappa v_{0}}\right) \operatorname{Re} F_{01}^{R}\right] \frac{\sqrt{1-p^{2}}}{\gamma_{0}}$,

and

$\zeta_{i}(x)=\left[\frac{\beta}{2} \frac{1}{1+x^{2}} P_{N}+\left(\frac{2 \pi D}{\kappa v_{0}}\right) \operatorname{Im} F_{01}^{R}\right] \frac{\sqrt{1-p^{2}}}{\gamma_{0}}$.

In the same case, the absorption coefficient (47) becomes

$\mu(x)=\mu_{N}(x)+\mu_{e}$,

where the absorption coefficients of $\gamma$-quanta by nuclei and electrons are defined by the expressions

$\mu_{N}(x)=\frac{\beta / D}{1+x^{2}}$

and

$\mu_{e}=\frac{1}{v_{0}} \sum_{j} \sigma_{e}^{(j)}$

respectively.

\section{Discussion}

We have generalized Kato's theory [8-11] for the Laue diffraction of the so-called spherical $\mathrm{x}$-ray waves to the case of Mössbauer diffraction. The analysis is confined by the symmetric Laue diffraction. Following [20], we have described the $\gamma$-photons by the wave packets $\boldsymbol{\Psi}(\mathbf{r} t)$ of Bialynicki-Birula [13] and Sipe [14]. They are formed by the plane waves $\chi_{\kappa s}(\mathbf{r}, t)$, which are spread over the frequency $\omega$ and angle $\theta$. By assumption, the wave vector $\boldsymbol{\kappa}_{0}$ corresponding to the distribution maximum exactly satisfies the Bragg condition specifying a two-wave diffraction. Each such component $\chi_{\kappa s}(\mathbf{r}, t)$ is scattered in a crystal independently of one another (see also [8-11]).

Kato's theory is based on the stationary-phase approximation, which is valid, if the phase $S_{\iota}(\eta)$ in the 

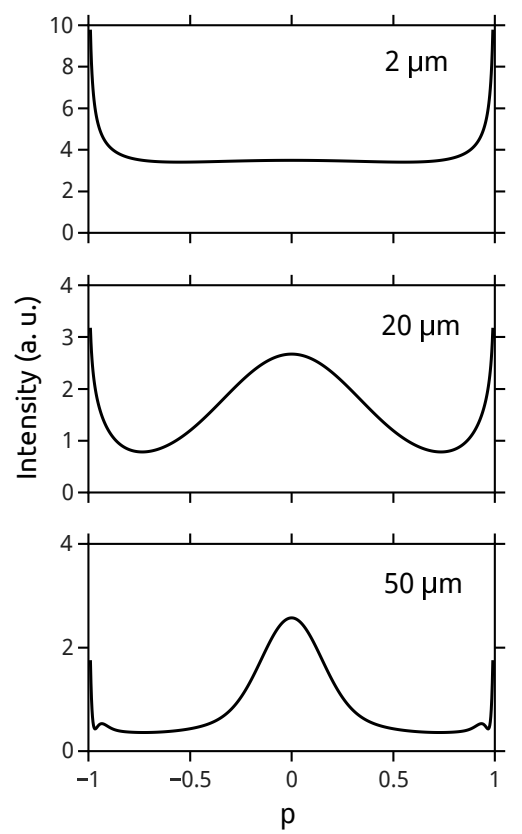

Fig. 2. Distribution of the intensity $I_{1}(p)$ of a Mössbauer beam diffracted in a tin film of various thicknesses over the Borrmann triangle as $-1<p<1$. The symmetric Laue diffraction is treated here, proceeding at the Bragg angle $\theta_{\mathrm{B}}=5^{\circ} 6^{\prime}$, exact resonance $\omega_{0}=\omega_{0}^{\prime}$, and the $100-\%$ abundance by the Mössbauer isotope ${ }^{119} \mathrm{Sn}$

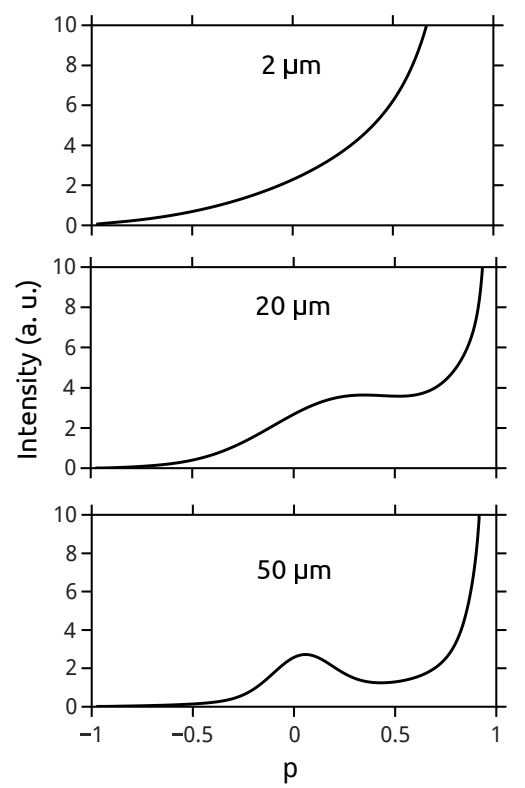

Fig. 3. The same as in Fig. 2, but for the refracted beam $I_{0}(p)$

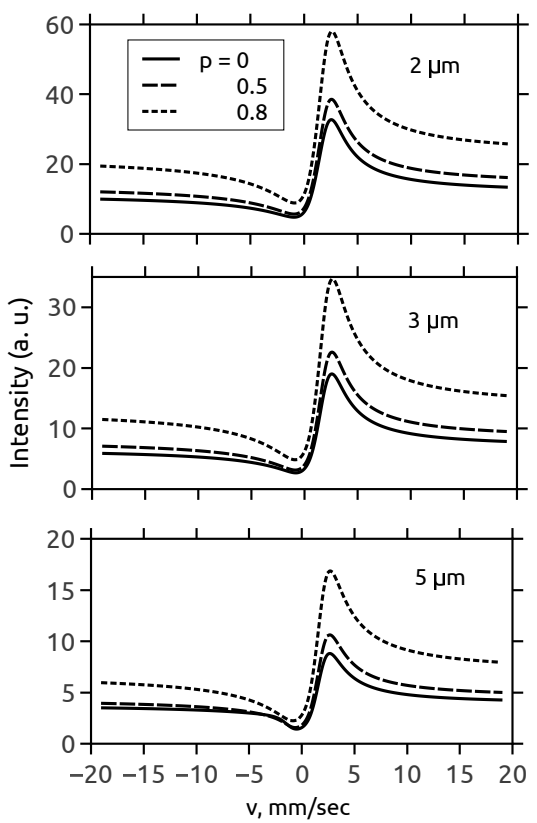

Fig. 4. Mössbauer spectrum of a diffracted beam at $p=0$ versus the relative motion velocity of the Mössbauer source and the target

exponential of integral (32) is a real function, i.e. if the absorption is practically absent. Instead, we use the more adequate saddle-point method for the evaluation of this integral in thick strongly absorbing crystals.

We have assumed the nuclear sublevels to be unsplit, which simplifies the consideration of polarizations. In particular, this is realized in nuclei ${ }^{119} \mathrm{Sn}$ with M1 transitions and the transition energy $E_{0}=$ $=23.8 \mathrm{keV}$. The Mössbauer diffraction in a tin single crystal was observed by Voitovetskii et al. [2325], while studying the suppression of inelastic channels and reactions predicted by Kagan and Afanas'ev [4]. We performed numerical calculations for the symmetric Laue diffraction in a tin crystal film (see Figs. 2-5), by choosing the same parameters as in one of the experiments reported in [23]. Namely, we consider the first-order reflection by the $(020)$ planes with the Bragg angle $\theta_{\mathrm{B}}=5^{\circ} 6^{\prime}$. We put the temperature $T=110 \mathrm{~K}$, when the ratio of the resonant nuclear amplitude $\left|f_{\text {res }}^{N}\right|$ to the Rayleigh one $f^{R}$ equals 3.2 , and the ratio of the absorption coefficients $\mu_{N} / \mu_{e}=167$ [23]. Taking into account that the Debye temperature of tin $\Theta_{D}=200 \mathrm{~K}$, we found the Lamb-Mössbauer factor to be $e^{-W}=0.48$ at $T=110 \mathrm{~K}$, which enabled 
us to get the $\left|f_{\text {res }}^{N}\right|$ by Eq.(12). All the curves drawn in Figs. 2-4 are calculated for the 100-\% abundance by ${ }^{119} \mathrm{Sn}$. The results presented in Figs. 2, 3, and 5 correspond to exact nuclear resonance, when $\omega_{0}=\omega_{0}^{\prime}$.

The calculated intensities of the diffracted beam $I_{1}(p)$ are shown in Fig. 2 as a function of the parameter $p$, ranging from -1 to +1 for the film thicknesses $D=2,20$, and $50 \mu \mathrm{m}$. The corresponding curves for the refracted beam are given in Fig. 3. Here, we see the same behavior as in the case of x-ray diffraction. Namely, in a thin weakly absorbing crystal, there is a growth of the diffracted intensity to the edges of the Borrmann triangle $p= \pm 1$. As the absorption grows with the film thickness, there appears a bump in the middle of the triangle $(p=0)$. In this case, the energy of $\gamma$-rays flows mainly along the reflecting planes, which explains a relative growth of the diffracted beam intensity at $p \approx 0$ in a strongly absorbing crystal (see also [1]).

The dependence of the diffracted beam intensity on the velocity of the Mössbauer emitter with respect to the species is analyzed in Fig. 4, where the thickness $D=2,3$, and $5 \mu \mathrm{m}$. The intensity curves apart from the peak manifest also a characteristic falloff caused by the interference of the waves scattered at the atomic electrons and the nucleus. Our results well correlate with the observations of Voitovetskii et al. [23].

Moreover, we have analyzed the dependence of the diffracted wave intensity $I_{1}(p)$ on the concentration of the Mössbauer isotope $c_{0}$. The results are displayed in Fig. 5 for the same tin crystal, but with the thickness $D=100 \mu \mathrm{m}$ and concentrations $c_{0}=0.5,0.1$, and 0 . We see that, with lowering $c_{0}$, there appears a fringe structure of the curve $I_{1}(p)$, which becomes most clear in the case $c_{0}=0$, corresponding to the pure Rayleigh scattering of Mössbauer radiation. With growing $c_{0}$, the oscillations of $I_{1}(p)$ arise only at the edges of the Borrmann triangle, while their amplitude decreases. For $c_{0}=1$, oscillations of the curves $I_{0,1}(p)$ are absent.

Such behavior has the following explanation resulting in a smooth dependence of the curve $I_{1}(p)$ versus the parameter $p$.

The diffracted wave packet (40) consists of the partial waves with definite frequencies $\omega$. Every such wave provides oscillations of the intensity through the function $f(x)=\sin ^{2}\left(\zeta_{r}(x)+\pi / 4\right)$, where $\zeta_{r}(x)$ is defined by Eq. (50). The distance between neighboring
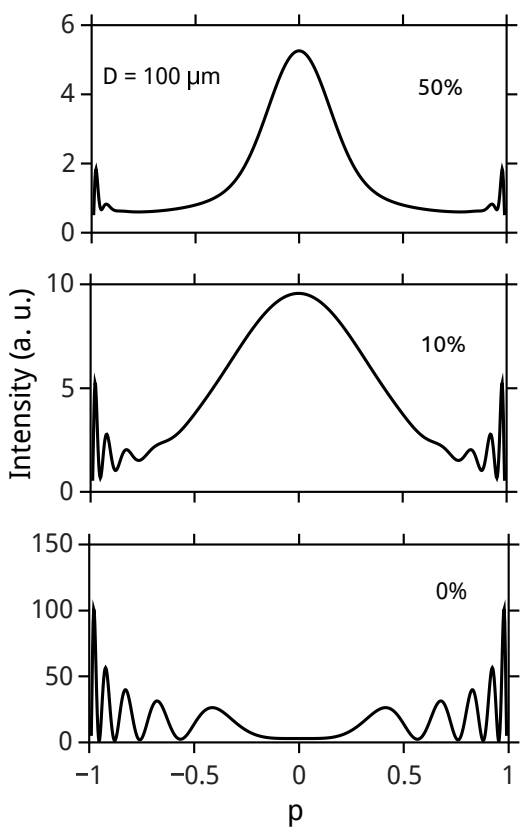

Fig. 5. Dependence of the intensity of diffracted radiation $I_{1}(p)$ on $p$ for concentrations of the Mössbauer isotope $c_{0}=$ $=0.5,0.1$, and 0

peaks of $I_{1}(p)$, depending on the frequency $\omega$, may be roughly estimated as

$\Delta p(x) \approx \frac{\pi\left(1-p^{2}\right) / p}{\sin \left(2 \zeta_{r}(x)+\pi / 2\right)\left|\zeta_{r}(x)\right|}$.

We see that it really diminishes at the edges of the Borrmann triangle, as $p \rightarrow \pm 1$. The quantity $\zeta_{r}(x)$ is determined by the sum of the Rayleigh scattering amplitude and the nuclear one. The latter is characterized by a sharp dependence on the frequency $\omega$. Since the coherent nuclear amplitude is proportional to $c_{0}$, the nuclear scattering prevails over the Rayleigh one at a sufficient abundance by the resonant isotope, and the averaging of the functions $f(x)$, having quite different periods of oscillations and located in integral (45), provides a smooth curve $I(p)$. Thus, in the experiments with the Rayleigh scattering of Mössbauer radiation, one can observe the intensity oscillations of the diffracted waves. Similar fringe oscillations have been observed by Shull $[26,27]$ in the famous neutron-optical experiments, which allowed him to measure the neutron-Si scattering length with high accuracy. One may hope that something like this can be also realized, by observing a fringe structure in the diffraction spectra of the Mössbauer or synchrotron radiation. 
1. B.W. Batterman and H. Cole, Dynamical diffraction of X rays by perfect crystals, Rev. Mod. Phys. 36, 681 (1964) [DOI: 10.1103/RevModPhys.36.681].

2. André Authier, Dynamical Theory of X-ray Diffraction (Oxford Univ. Press, New York, 2001).

3. Z.G. Pinsker, Dynamical Scattering of X-rays in Crystals (Springer, Heidelberg, 1978).

4. A.M. Afanas'ev and Yu. Kagan, Suppression of inelastic channels in resonant nuclear scattering in crystals, Sov. Phys. JETP 21 (1), 215 (1965).

5. Yu. Kagan and A.M. Afanas'ev, Suppression of inelastic channels in resonance scattering of neutrons in regular crystals Sov. Phys. JETP 22, 1032 (1966).

6. J.P. Hannon and G.T. Trammell, Mössbauer diffraction. I. Quantum theory of gamma-ray and X-ray optics, Phys. Rev. 169, 315 (1968).

7. J.P. Hannon and G.T. Trammell, Mössbauer diffraction. II. Dynamical theory of Mössbauer optics, Phys. Rev. 186, 306 (1969) [DOI: 10.1103/PhysRev.186.306].

8. N. Kato, The energy flow of X-rays in an ideally perfect crystal: comparison between theory and experiments, Acta Cryst. 13, 349 (1960) [DOI: 10.1107/S0365110X60000819].

9. N. Kato, A theoretical study of pendellosung fringes. I. General considerations, Acta Cryst. 14, 526 (1961) [DOI: 10.1107/S0365110X61001625].

10. N. Kato, A theoretical study of pendellosung fringes. II. Detailed discussion based upon a spherical wave theory, Acta Cryst. 14, 627 (1961) [DOI: 10.1107/ S0365110X61001947].

11. N. Kato, J. Phys. Soc. Japan 19, 971 (1964).

12. V.B. Berestetskii, E.M. Lifshitz, and L.P. Pitaevskii, Quantum Electrodynamics (Pergamon, Oxford, 1982).

13. I. Bialynicki-Birula, On the wave function of the photon, Acta Phys. Pol. 86, 97 (1994).

14. J.E. Sipe, Photon wave functions, Phys. Rev. A 52, 1875 (1995).

15. I. Bialynicki-Birula, Photon wave functions, Progr. Opt. 36, 245 (1996).

16. R.J. Smith and M.G. Raymer, Photon wave functions, wave-packet quantization of light and coherence theory, New J. Phys. 9, 414 (2007) [DOI: 10.1088/13672630/9/11/414].

17. P.J. Mohr, Solutions of the Maxwell equations and photon wave functions, Ann. Phys. 325, 607 (2010) [DOI: 10.1016/j.aop.2009.11.007].
18. J. Cugnon, The photon wave function, Open J. Microphys. 1, 41 (2011) [DOI: 10.4236/ojm.2011.13008].

19. N. Chandrasekar, Quantum mechanics of photons, Adv. Studies Theor. Phys. 6, 391 (2012).

20. A.Ya. Dzyublik, NPAE 13, No. 2 (2015).

21. V.A. Belyakov, Diffraction of Mössbauer gamma rays in crystals, Sov. Phys. USP 18, 267 (1975).

22. M.A. Lavrentiev and B.V. Shabat, Methods of the Theory of Functions of Complex Variable (Nauka, Moscow, 1973) (in Russian).

23. V.K. Voitovetskii et al., Diffraction of resonance $\gamma$-rays by nuclei and electrons in tin single crystals, Sov. Phys. JETP 27, 729 (1968).

24. V.K. Voitovetskii et al., Experimental evidence of disappearance of the inelastic channel of the nuclear reaction in the interaction of resonant $\gamma$-radiation with nuclei and electrons in a single crystal, Phys. Lett. A 28, 779 (1969) [DOI: 10.1016/0375-9601(69)90619-7].

25. V.K. Voitovetskii et al., Observation of the suppression of the inelastic channel of a nuclear reaction in resonant nuclear scattering of gamma-rays in a perfect single crystal, JETP Lett. 11, 91 (1970).

26. C.G. Shull, Observation of pendellosung fringe structure in neutron diffraction, Phys. Rev. Lett. 21, 1585 (1968) [DOI: 10.1103/PhysRevLett.21.1585].

27. C.G. Shull, Perfect crystals and imperfect neutrons, J. Appl. Cryst. 6, 257 (1973) [DOI: 10.1107/ $\mathrm{S} 0021889873008654]$.

Received 20.03.16

О.Я. Дзюблик, В.Ю. Співак

ДИФРАКЦІЯ ЛАУЕ

МЕССБАУЕРІВСЬКИХ СФЕРИЧНИХ ХВИЛЬ

$\mathrm{P}$ е $з$ ю м е

В наближенні сферичних хвиль аналізується симетрична дифракція Лауе мессбауерівських променів. Для обчислення хвильової функцї гамма-фотонів у межах трикутника Бормана в товстому кристалі із сильним ядерним поглинанням використовується метод перевалу. Враховується як релеївське, так і резонансне ядерне розсіяння. Показано, що у випадку релеївського розсіяння мессбауерівського випромінювання виникають інтерференційні осциляції інтенсивності дифрагованого пучка, які можна використовувати для прецизійних вимірювань параметрів кристала. 